\title{
COMPLETELY CONTINUOUS TRANSFORMATIONS IN HILBERT SPACE
}

\section{F. SMITHIES}

Let $\mathfrak{S}$ be a real or complex Hilbert space; we do not assume that $\mathfrak{S}$ is separable. The following theorem is well known:*

THEOREM. Let $T$ be a linear symmetric completely continuous transformation defined throughout $\mathfrak{S}$ and not identically zero. Then there exist an element $x$ of $\mathfrak{S}$ and a real number $\lambda$ such that $x \neq 0, \lambda \neq 0$, and $T x=\lambda x$.

The proof presented below is rather simpler than Rellich's, though based on the same fundamental idea.

We first state a familiar lemma.

LEMma. $\dagger$ If $T$ is a linear symmetric transformation defined throughout $\mathfrak{S}$ such that $|(T x, x)| \leqq A$ whenever $\|x\|=1$, then $\|T x\| \leqq A$ whenever $\|x\|=1$.

The range of values of the expression $(T x, x)$, for elements $x$ such that $\|x\|=1$, is a bounded set of real numbers. For some such $x$, $(T x, x) \neq 0$; if not, by the lemma, $T$ would vanish identically. Let

$$
\lambda=\sup |(T x, x)|, \quad\|x\|=1 .
$$

Without loss of generality, we may suppose that

$$
0<\lambda=\sup (T x, x), \quad\|x\|=1 .
$$

Otherwise we consider $-T$ instead of $T$. We can choose $\left\{x_{n}\right\}$ such that $\left\|x_{n}\right\|=1,(n=1,2, \cdots)$, and $\left(T x_{n}, x_{n}\right) \rightarrow \lambda, n \rightarrow \infty$. Since $T$ is completely continuous, $\left\{T x_{n}\right\}$ contains a convergent subsequence, which we may take to be $\left\{T x_{n}\right\}$ itself. Suppose that $T x_{n} \rightarrow y$. Then because $T$ is symmetric and $\left\|x_{n}\right\|=1$,

$$
\left\|T x_{n}-\lambda x_{n}\right\|^{2}=\left\|T x_{n}\right\|^{2}-2 \lambda\left(T x_{n}, x_{n}\right)+\lambda^{2} .
$$

Hence, letting $n \rightarrow \infty$, we have

$$
0 \leqq \lim _{n \rightarrow \infty}\left\|T x_{n}-\lambda x_{n}\right\|^{2}=\|y\|^{2}-\lambda^{2} .
$$

* F. Rellich, Spektraltheorie in nichtseparabeln Räumen, Mathematische Annalen, vol. 110 (1934), pp. 342-356; 348.

$\dagger$ For a proof of this lemma, see M. H. Stone, Linear Transformations in Hilbert Space and their Applications to Analysis, American Mathematical Society Colloquium Publications, vol. 15, New York, 1932, pp. 54-56. 
By (1) and the lemma, $\left\|T x_{n}\right\| \leqq \lambda,(n=1,2, \cdots)$, so that $\|y\| \leqq \lambda$. It therefore follows from (2) that

$$
\lim _{n \rightarrow \infty}\left\|T x_{n}-\lambda x_{n}\right\|^{2}=0 .
$$

Now $T x_{n} \rightarrow y$; hence by (3), $\lambda x_{n} \rightarrow y$. Since $\lambda \neq 0$ and $T$ is continuous, it follows that $T x_{n} \rightarrow T y / \lambda$, so that $T y=\lambda y$. Finally, by (2) and (3), $\|y\|=\lambda \not 0$, and the theorem is therefore proved.

INSTITUTE for AdVANCEd StUdy

\section{UN PROBLEMME DE LA THEORIE DES NOMBRES RATTACHE AUX POLYNÔMES DE TSCHEBYCHEFF}

\section{ERVIN FELDHEIM}

Considérons le polynôme de Tschebycheff particulier

$$
\begin{aligned}
& B_{n}(x)=\frac{\left[x+\left(x^{2}+4\right)^{1 / 2}\right]^{n+1}-\left[x-\left(x^{2}+4\right)^{1 / 2}\right]^{n+1}}{2^{n+1}\left(x^{2}+4\right)^{1 / 2}}, \\
& n=-1,0,1,2, \cdots .
\end{aligned}
$$

Si l'on donne à la variable $x$ la valeur $x=2$, ce polynôme prend, comme il est très facile de le vérifier, des valeurs entières, et la suite de ces nombres entiers possède des propriétés intéressañtes que nous nous proposons de démontrer dans cette note.

Nous déduirons d'abord quelques relations valables pour les polynômes $B_{n}(x)$; la propriété des nombres mentionnés tout à l'heure que nous voulons établir s'en résultera facilement. Nous écrivons, pour simplifier, $B_{n}$ au lieu de $B_{n}(x)$, de sorte que $B_{n}$ désigne toujours un polynôme et non pas un nombre; pour $x=2$ nous introduirons une nouvelle notation.

La relation principale qui nous servira est bien connue, et se démontre d'ailleurs facilement en partant de (1). C'est la relation

$$
B_{n+m}=B_{n} B_{m}+B_{n-1} B_{m-1}, \quad n, m=0,1,2, \cdots .
$$

En tenant compte des valeurs initiales du polynôme $B_{n}$, calculées au moyen de (1),

$$
B_{-1}=0, \quad B_{0}=1, \quad B_{1}=x, \quad B_{2}=x^{2}+1,
$$

on déduit de (2) une série de relations utiles. On a d'abord 